\title{
Contingent tolerance and cross-tolerance to anticonvulsant drug effects: Pentobarbital and ethanol
}

\author{
JOHN P. J. PINEL, C. KWON KIM, DENNIS J. PAUL, and MICHAEL J. MANA \\ University of British Columbia, Vancouver, British Columbia, Canada
}

\begin{abstract}
Tolerance to the anticonvulsant effect of pentobarbital $(15 \mathrm{mg} / \mathrm{kg}$, i.p.) was developed in amygdala-kindled rats; it was found to be contingent upon the rats' receiving convulsive stimulations during the bidaily (once every $48 \mathrm{~h}$ ) periods of pentobarbital exposure (Experiment 1B; contingent tolerance). Furthermore, the subjects that received convulsive stimulations while un der the influence of the bidaily pentobarbital injections subsequently displayed a greater degree of cross-tolerance to the anticonvulsant effect of $1.5 \mathrm{~g} / \mathrm{kg}$ of ethanol than those that had received the drug after the stimulations (Experiment 2; contingent cross-tolerance). Finally, contingent tolerance to the anticonvulsant effect of ethanol was maintained when kindled rats received convulsive stimulations during, but not before, bidaily periods of pentobarbital exposure (Experiment 3; contingent cross maintenance of tolerance). These results suggest that the repeated manifestation of particular drug effects-anticonvulsant effects in this case-play an important role in the development of some forms of drug tolerance and in their transfer to other drugs.
\end{abstract}

Contingent drug tolerance (Carlton \& Wolgin, 1971) is drug tolerance that is contingent on the repeated expression of the drug effect rather than on mere exposure to the drug. Most demonstrations of contingent drug tolerance (sometimes ambiguously referred to as "behavioral tolerance") have used the before-and-after design (Kumar \& Stolerman, 1977). With this design, the subjects in one group (the drug-before group) receive the drug $b e$ fore they perform the test response on each tolerancedevelopment trial, whereas the subjects in the other group (the drug-after group) do not receive the drug on each trial until after they have performed the test response. Thus, the subjects in the drug-before group experience the drug's effect on the test response on each trial, whereas those in the drug-after group do not. Then, on the tolerance-test trial, the subjects in both groups receive the drug before they perform the test response, to assess the degree to which tolerance has developed in each. Because the drug-before and the drug-after subjects are exposed to exactly the same regimen of drug injections, evidence of greater tolerance in the drug-before subjects is attributed to the difference in the contingency between the drug exposure and the test response.

Many examples of drug tolerance have been shown to be influenced by the response contingency (for a review, see Demellweek \& Goudie, 1983). For example, toler-

This research was supported by a grant from the Medical Research Council (MRC) to the senior author, and by an MRC postgraduate scholarship to Michael J. Mana. We wish to acknowledge the helpful comments of Craig Hilton Jones. All correspondence may be addressed to J. P. J. Pinel, Department of Psychology, University of British Columbia, Vancouver, British Columbia V6T 1Y7, Canada. ance to the anorexigenic effect of amphetamine is greater in rats allowed to feed while drugged (Carlton \& Wolgin, 1971); tolerance to the disruptive effect of ethanol on circular-maze running is greater in rats allowed to run the maze while intoxicated (Chen, 1968); and tolerance to the accelerating effect of ethanol on the decay of posttetanic potentiation in the abdominal ganglion of Aplysia develops only if the presynaptic terminal is repeatedly stimulated in the presence of ethanol (Traynor, Schlapfer, \& Barondes, 1980).

This report comprises a series of four experiments, each conducted on the same sample of rats and each designed to study contingent tolerance to the anticonvulsant effects of sodium pentobarbital (pentobarbital) and ethanol on kindled convulsions.

\section{GENERAL METHOD}

\section{Subjects}

The subjects in the four experiments were 30 adult male, 440-540-g hooded Long-Evans rats obtained from Charles River, Canada. The subjects were individually housed in standard stainless steel hanging cages in a room with an ambient temperature of about $21^{\circ} \mathrm{C}$ and a $12: 12$-h light:dark cycle. All experimental manipulations occurred during the light phase of the cycle. Purina Rat Chow and water were continuously available in the home cages.

\section{Surgery}

Following the administration of sodium pentobarbital $(65 \mathrm{mg} / \mathrm{kg}$, i.p.) and atropine sulphate $(0.04 \mathrm{mg}$, i.p. $)$, a single chronic bipolar electrode (Plastic Products Company, MS 303/2) was directed at the basolateral amygdala of each of 30 rats. It was secured to the skull with stainless steel screws and dental acrylic. The tip of each electrode was positioned $1.2 \mathrm{~mm}$ posterior, $5.0 \mathrm{~mm}$ lateral, and $10.0 \mathrm{~mm}$ ventral to the skull surface at bregma, with the incisor 
bar set at $+5.0 \mathrm{~mm}$ (coordinates from Pellegrino, Pellegrino, \& Cushman, 1979). Tetracycline was sprinkled over the incision before suturing.

\section{Kindling Phase}

Beginning at least 5 days following surgery, each of the 30 rats was stimulated $(400 \mu \mathrm{A} \mathrm{rms}, 60 \mathrm{~Hz}, 1 \mathrm{sec})$ three times per day, 5 days a week for 3 weeks, with at least $2 \mathrm{~h}$ separating consecutive stimulations. At first the stimulations produced no behavioral response, but by the end of the regimen of 45 kindling stimulations, each produced a generalized clonic seizure (cf. Pinel \& Rovner, 1978; Racine, 1972). The measure of seizure severity was the duration of forelimb clonus elicited by each stimulation.

\section{Treatment and Testing Phases}

The treatment and testing phases of the four experiments were different, but the bidaily (once every $48 \mathrm{~h}$ ) stimulation regimen initiated following the initial kindling phase was maintained without interruption throughout all four.

\section{Histology}

At the conclusion of the last experiment, all surviving subjects were killed with $\mathrm{CO}_{2}$ according to the Canada Council on Animal Care guidelines and perfused intracardially with $4 \%$ formalin. Their brains were removed, preserved in formalin, frozen, sliced along the coronal plane (30- $\mu \mathrm{m}$ thickness), mounted on slides, and then stained with cresyl violet to confirm the locations of the electrode tips. Each of the electrodes terminated in the amygdaloid complex, the majority within the basolateral nucleus.

\section{EXPERIMENT 1A}

Experiment 1A had two purposes: (1) to demonstrate that tolerance to the anticonvulsant effect of pentobarbital develops, and (2) to determine whether or not tolerance to the anticonvulsant effect of pentobarbital is contingent on the occurrence of convulsive stimulations during the periods of pentobarbital exposure.

\section{Method}

The baseline and screening phase of Experiment 1A began $48 \mathrm{~h}$ after the last of the 45 kindling stimulations, and it involved five stimulations delivered once every $48 \mathrm{~h}( \pm 3 \mathrm{~h})$. The isotonic-saline vehicle was injected $1 \mathrm{~h}$ before the fourth baseline stimulation (the saline baseline test). The injection volume for saline and all subsequent injections of pentobarbital (M.T.C. Pharmaceutical, Mississauga, Ontario) was $7.5 \mathrm{ml} / \mathrm{kg}$, i.p.. This volume was chosen to match the volume of ethanol delivered in Experiments 2 and 3: i.p. ethanol must be administered in low concentrations to minimize distress to the rats. A drug baseline test was administered on the 5 th baseline day; all the subjects received pentobarbital $(20 \mathrm{mg} / \mathrm{kg}$, i.p.) $1 \mathrm{~h}$ before stimulation. Six rats were dropped from the experiment during the baseline phase: 1 became ill, 1 lost its electrode assembly, 1 did not meet the criterion of at least $20 \mathrm{sec}$ of forelimb clonus on the saline baseline test, and 3 displayed running fits, which are violent, variable, and difficult to score.

The treatment phase of Experiment 1A began $48 \mathrm{~h}$ after the drug baseline test; it comprised 10 bidaily stimulations and 10 bidaily injections. The 24 rats beginning Experiment $1 \mathrm{~A}$ were divided into two equivalent groups on the basis of the durations of their forelimb clonus on the saline and drug baseline tests and their body weights. On each treatment session, the rats in one group (the pentobarbital-before-stimulation group; $n=12$ ) received pentobarbital $(20 \mathrm{mg} / \mathrm{kg}$, i.p.) $1 \mathrm{~h}$ before the convulsive stimulation, and the rats in the other group (the pentobarbital-after-stimulation group; $n=12$ ) received pentobarbital $1 \mathrm{~h}$ after the stimulation.
The posttreatment tolerance test occurred $48 \mathrm{~h}$ after the $10 \mathrm{th}$ and last treatment session. This time, each rat received $20 \mathrm{mg} / \mathrm{kg}$ of pentobarbital $1 \mathrm{~h}$ before the stimulation, so that the development of tolerance to pentobarbital's anticonvulsant effect could be assessed.

\section{Results}

It is clear from Panel A of Figure 1 that there was no evidence of tolerance to the anticonvulsant effect of $20 \mathrm{mg} / \mathrm{kg}$ of pentobarbital in either experimental group. The ability of $20 \mathrm{mg} / \mathrm{kg}$ of pentobarbital to block kindled seizures was not significantly reduced by the 10 treatment injections in either the pentobarbital-before-stimulation group (sign test: $N=3, X=0, p>.01$ ) or the pentobarbital-after-stimulation group (sign test: $N=4$, $X=2, p>.01$ ). Accordingly, the difference between the two groups on the tolerance test $[U(12,12)=68.5$, $p>.01]$ was not significant.

\section{EXPERIMENT 1B}

It seemed premature to conclude that tolerance to the anticonvulsant effect of pentobarbital does not develop, in view of reports of tolerance to the anticonvulsant effects of ethanol (e.g., Allan \& Swinyard, 1949; Pinel, Colbourne, Sigalet, \& Renfrey, 1983), which has a similar anticonvulsant profile to that of pentobarbital, and reports of tolerance to the anticonvulsant effect of phenobarbital (e.g., Frey \& Kampmann, 1965), which has a pharmacological profile similar to that of pentobarbital (see Prichard, 1980). In Experiment 1B, we demonstrated that tolerance to the anticonvulsant effect of pentobarbital does indeed develop, and that its development is contingent on the occurrence of convulsive stimulation during the periods of pentobarbital exposure.

\section{Method}

There were two differences between the methods of Experiments $1 \mathrm{~A}$ and $1 \mathrm{~B}$, both designed to increase the likelihood of detecting the hypothesized tolerance effect. The dose of pentobarbital was reduced from 20 to $15 \mathrm{mg} / \mathrm{kg}$ (in a $7.5 \mathrm{ml} / \mathrm{kg}$ volume of saline, i.p.), and the number of tolerance development trials was increased from 10 to 15 .

Experiment $1 \mathrm{~B}$ commenced $48 \mathrm{~h}$ after the tolerance test in Experiment 1A. It began, as had Experiment 1A, with the five baseline stimulations, and again the fourth and fifth of these baseline stimulations constituted saline and drug baseline tests, respectively. Of the 24 rats that began Experiment 1B, one displayed running fits during the baseline phase and was not studied further. The remaining 23 rats were equally $( \pm 1)$ distributed from the two groups from Experiment $1 \mathrm{~A}$ to the pentobarbital-before-stimulation $(n=12)$ and the pentobarbital-after-stimulation $(n=11)$ groups of Experiment 1B, in such a way that the mean durations of forelimb clonus elicited in the two groups on the saline and drug baseline tests of Experiment IB and their weights were comparable.

\section{Results and Discussion}

The results of Experiment 1B are illustrated in Panel B of Figure 1. The decreasing efficacy with which the pentobarbital blocked the clonus produced by amygdaloid stimulation in the pentobarbital-before-stimulation condition is readily apparent. On the drug baseline test, 


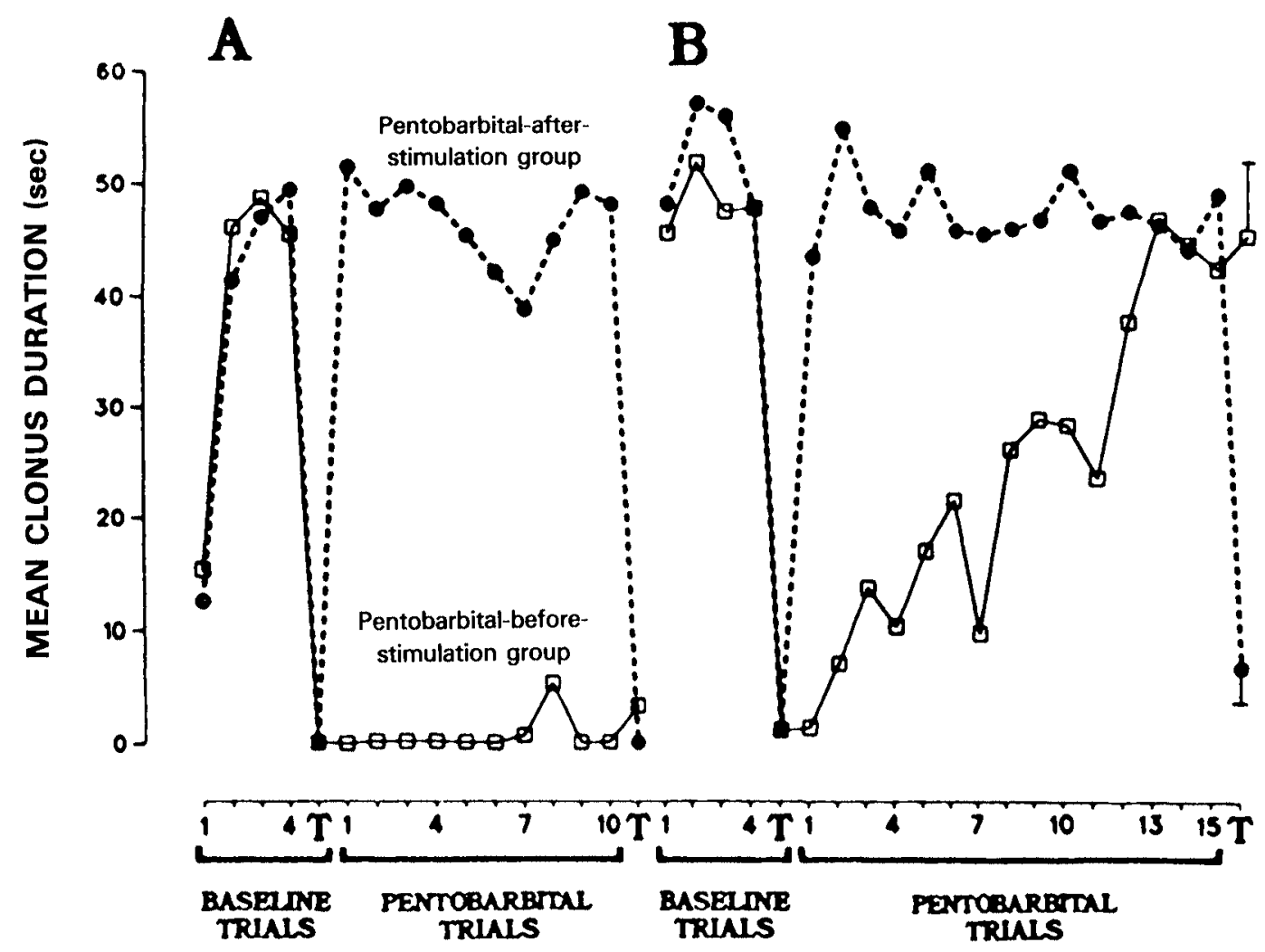

Figure 1. The effect of pentobarbital administration either before or after convulsive stimulations. Tolerance was not apparent after $1020-\mathrm{mg} / \mathrm{kg}$ bidaily treatment injections in either the pentobarbital-before-stimulation or the pentobarbital-after-stimulation conditions in Experiment 1A (Panel A). Tolerance was clearly apparent after 15 15$\mathrm{mg} / \mathrm{kg}$ bidaily treatment injections in Experiment $1 \mathrm{~B}$ (Panel B), but only in the pentobarbital-before-stimulation condition. Error bars indicate the standard error on test days: note the total lack of variability in some conditions.

pentobarbital totally suppressed forelimb clonus in almost every pentobarbital-before-stimulation rat, whereas, on the final test day, after the 15 treatment injections, it had no apparent anticonvulsant effect (sign test: $N=12$, $X=0, p<.002$ ). In contrast, there was little evidence of tolerance in the pentobarbital-after-stimulation condition. Pentobarbital totally suppressed the forelimb clonus of the pentobarbital-after-stimulation rats on the drug baseline test; and it suppressed it again on the posttreatment tolerance test, albeit to a slightly lesser degree (sign test: $N=8, X=1, p>.01$ ). Accordingly, although the rats in both groups received the same number of drug injections and convulsive stimulations, there was a striking difference between them on the posttreatment tolerance test $[U(11,12)=4, p<.001]$. The results of Experiment 1B thus confirm the two main hypotheses: first, that tolerance to the anticonvulsant effect of pentobarbital does indeed develop, and second, that its development is contingent on the occurrence of convulsive stimulation during the periods of pentobarbital exposure.

In view of the robust contingent tolerance effects in Experiment 1B, their absence in Experiment $1 \mathrm{~A}$ requires comment. It is possible that pentobarbital injected at $15 \mathrm{mg} / \mathrm{kg}$ produces large tolerance effects that are not produced at all by $20-\mathrm{mg} / \mathrm{kg}$ injections, but we favor the view that tolerance was developing in Experiment $1 \mathrm{~A}$ but was not reflected in a significant increase in clonus duration because $20 \mathrm{mg} / \mathrm{kg}$ was well above the threshold dose for complete clonus suppression. This view is supported by the observation of Pinel et al. (1983) that tolerance to the anticonvulsant effect of large doses of ethanol was not apparent until both the ethanol and vehicle control rats were challenged by a smaller test dose. Inspection of Panel B of Figure 1 suggests that this problem may have been compounded by the fact that the tolerance to the anticonvulsant effect of pentobarbital is not asymptotic after 10 bidaily injections; perhaps tolerance would have eventually been observed in Experiment $1 \mathrm{~A}$, even at the 20- $\mathrm{mg} / \mathrm{kg}$ dose, if more injections had been administered.

Although the decrease in the degree to which pentobarbital suppressed the seizures of the pentobarbital-afterstimulation rats during the course of the experiment did not achieve statistical significance, it warrants comment because it duplicates the results observed in some of our studies of contingent tolerance to the anticonvulsant effect of ethanol (e.g., Pinel, Mana, \& Renfrey, 1985). In several of our previous experiments, there has been a suggestion-albeit an insignificant one-of tolerance development in the ethanol-after-stimulation condition. Accordingly, although there is no question that the 
response contingency is extremely important for the development of tolerance to anticonvulsant drug effects, it is premature to conclude that it is essential.

\section{EXPERIMENT 2}

Does the response contingency influence the transfer of tolerance to anticonvulsant effects between drugs? The purpose of Experiment 2 was to determine whether the rats that had been exposed to pentobarbital under the pentobarbital-before-stimulation condition in Experiment $1 \mathrm{~B}$ would prove more tolerant to the anticonvulsant effect of ethanol than would those of the pentobarbitalafter-stimulation condition. There had been only two previous reports of contingent cross-tolerance: both were studies of the transfer between ethanol and pentobarbital of contingent tolerance to their effects on motor coordination and balance (Commissaris \& Rech, 1981; Lê, El-Ghundi, Khanna, \& Kalant, 1986).

\section{Method}

The two groups of subjects from Experiment 1B-the pentobarbital-before-stimulation group $(n=12)$, which had developed tolerance to the anticonvulsant effect of pentobarbital, and the pentobarbital-after-stimulation group $(n=11)$, which had notremained intact for Experiment 2. The subjects in each of these two groups received ethanol $(1.5 \mathrm{~g} / \mathrm{kg}$ in a $7.5-\mathrm{ml} / \mathrm{kg}$ volume of $25 \%$ $\mathrm{V} / \mathrm{V}$ solution of saline, i.p.) $1 \mathrm{~h}$ before each of 16 bidaily convulsive stimulations. This dose of ethanol was chosen because previous research had shown that it would block kindled seizures and that tolerance to this effect would develop (e.g., Pinel \& Mana, 1986).

The contingent cross-tolerance of the subjects in the two groups was assessed in two different ways-by the ability of the first injection of ethanol to suppress forelimb clonus, and by the rate at which the criterion of tolerance to ethanol's anticonvulsant effect was achieved. The criterion was forelimb clonus of at least 20 -sec duration on two consecutive trials.

\section{Results and Discussion}

Figure 2 shows that the rats that had been exposed to a regimen of pentobarbital-before-stimulation treatments in Experiment 1B were more tolerant to the first injection of ethanol than were the rats that had been exposed to the pentobarbital-after-stimulation treatments. The first ethanol injection completely suppressed the forelimb clonus of almost every pentobarbital-after-stimulation rat, whereas most of the rats in the pentobarbital-beforestimulation group demonstrated a substantial amount of forelimb clonus $[U(11,12)=0, p<.001]$. Moreover, the pentobarbital-before-stimulation rats reached the criterion of tolerance in a mean of only 3.1 ethanol injections in comparison to the 8.5 injections required by the pentobarbital-after-stimulation rats $[U(11,12)=2$, $p<.001]$. One rat in the pentobarbital-after-stimulation condition did not achieve the criterion, and it was arbitrarily assigned a score of 16 , the total number of ethanol injections, for the purpose of calculating the group means. On the 16th and final day of the experiment, the groups did not differ significantly $[U(11,12)=44, p>.01]$.

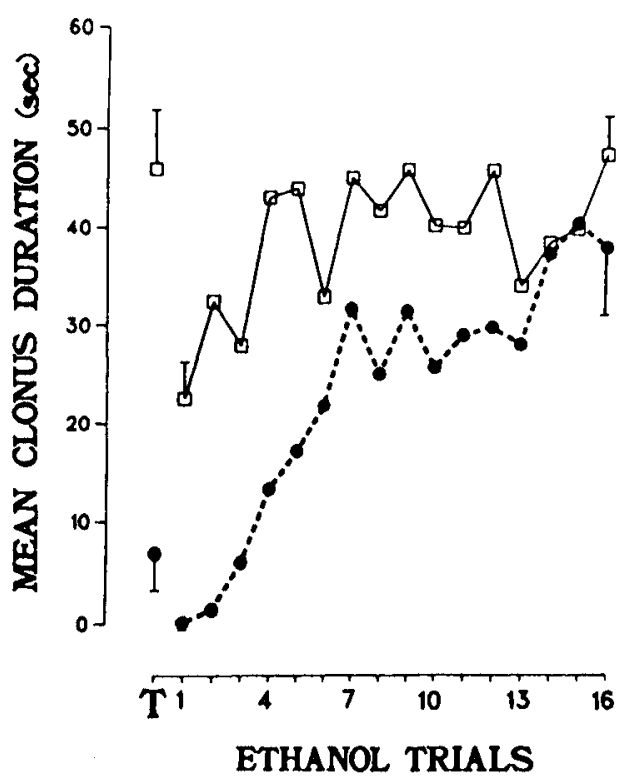

Figure 2. The effect of the response contingency on the transfer of tolerance from the anticonvulsant effect of pentobarbital to ethanol. The rats in both groups received injections of ethanol $(1.5 \mathrm{~g} / \mathrm{kg}) 1 \mathrm{~h}$ before each of the 16 bidaily convulsive stimulations. The subjects in one group (squares) had been rendered tolerant to pentobarbital in Experiment $1 \mathrm{~B}$ by a regimen of pentobarbitalbefore-stimulation treatments; those in the other group (circles) had been exposed to the same injections of pentobarbital on a pentobarbital-after-stimulation regimen. Error bars indicate the standard error on test days.

By demonstrating the generalization of contingent tolerance to the anticonvulsant effects of pentobarbital to the anticonvulsant effects of ethanol, Experiment 2 confirms and extends the original reports of Commissaris and Rech (1981) and Lê et al. (1986). They confirm that the response contingency can be a major factor in crosstolerance, and they establish for the first time that contingent cross-tolerance is not limited to the disruptive effects of drugs on balance and motor coordination.

\section{EXPERIMENT 3}

The observation of contingent cross-tolerance in Experiment 2 considered in combination with the recent report of Mana and Pinel (1987) that the response contingency also plays a major role in the dissipation of tolerance suggested an interesting possibility. Mana and Pinel found that a noncontingent schedule (i.e., ethanol-afterstimulation schedule) not only did not lead to the development of tolerance to the anticonvulsant effect of ethanol, it actually caused tolerance that had already been produced on an ethanol-before-stimulation schedule to dissipate. The purpose of Experiment 3 was to demonstrate that manipulating the response contingency during a series of pentobarbital injections can influence the maintenance and dissipation of a previously developed tolerance to the anticonvulsant effect of ethanol. We hypothesized that a pentobarbital-before-stimulation regimen would maintain 
the developed tolerance to the anticonvulsant effect of ethanol, but that a comparable regimen of pentobarbitalafter-stimulation injections would not.

\section{Method}

The 22 subjects completing Experiment 2 that had achieved the criterion of tolerance-two consecutive seizures with the forelimb clonus of $20 \mathrm{sec}$ or greater-were reassigned to two new groups so as to distribute subjects with differing experimental histories equally $( \pm 1)$ and to equate the two new groups for tolerance to ethanol on the last test session in Experiment 2. One of these 22 rats became ill during the course of Experiment 3, so its data were not subjected to analysis. Each subject in the two groups received six treatment injections of pentobarbital ( $15 \mathrm{mg} / \mathrm{kg}$, i.p.), once every $48 \mathrm{~h}$. The subjects in the pentobarbital-before-stimulation group $(n=11)$ received each injection $1 \mathrm{~h}$ before an amygdaloid stimulation, whereas those in the pentobarbital-after-stimulation group $(n=10)$ received each injection $1 \mathrm{~h}$ after a stimulation. On the last day of Experiment $3,48 \mathrm{~h}$ after the sixth pentobarbital treatment injection, the tolerance of all of the subjects to the anticonvulsant effect of ethanol was assessed by injecting them with ethanol $(1.5 \mathrm{~g} / \mathrm{kg}$, i.p.) $1 \mathrm{~h}$ before the convulsive stimulation.

\section{Results and Discussion}

The results of Experiment 3, summarized in Figure 3, were as predicted. The pentobarbital-after-stimulation

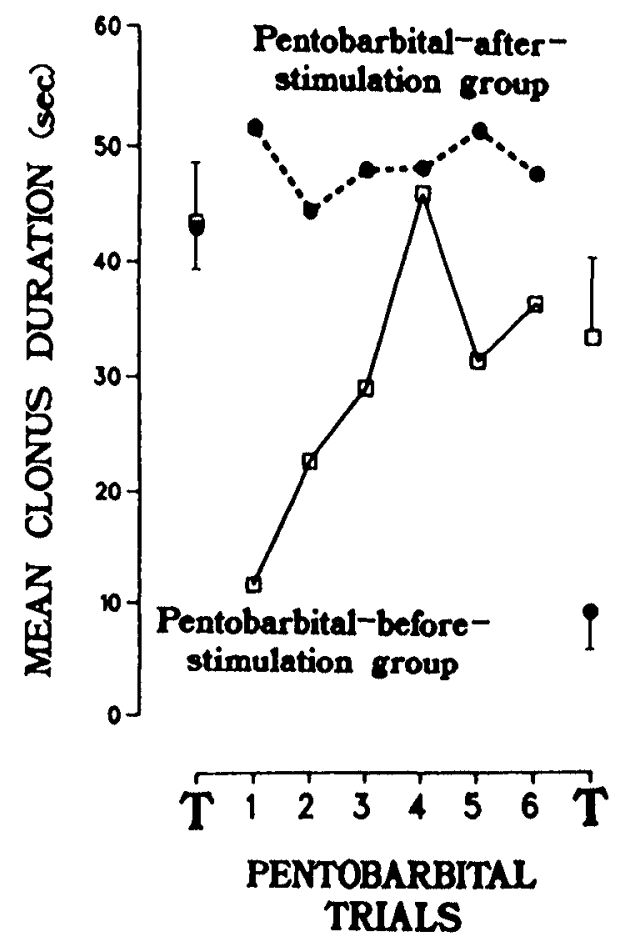

Figure 3. The dissipation or maintenance of tolerance to the anticonvulsant effect of ethanol after injections of pentobarbital either before or after the convulsive stimulations. The first points on the graph represent the initially equal degree of tolerance to ethanol that the two groups displayed on the last session of Experiment 2; the last points represent the responses of the two groups on the final ethanol tolerance test after the intervening pentobarbital treatments. The pentobarbital-after-stimulation treatments produced a significant decline in ethanol tolerance, but the pentobarbital-beforestimulation treatments did not. Error bars indicate the standard error on test days. regimen was associated with a significant decline in the duration of forelimb clonus between the last day of ethanol injection in Experiment 2 (the first points in Figure 3) and the ethanol test injection following the six pentobarbital injections in Experiment 3 (sign test: $N=10, X=0$, $p<.001)$, whereas the pentobarbital-before-stimulation regimen was not ( $\operatorname{sign}$ test: $N=11, X=5, p>.01$ ). Accordingly, the duration of the forelimb clonus elicited in the pentobarbital-before-stimulation group on the posttreatment ethanol test day was significantly greater than that elicited in the pentobarbital-after-stimulation group $[U(10,11)=15, p<.005]$.

The results of Experiment 3 demonstrate for the first time that one drug (pentobarbital) must manifest its criterion effect (its anticonvulsant effect in this case) to maintain tolerance to the same effect of another drug (ethanol), an effect that we call contingent cross maintenance of tolerance. However, Experiment 3 does not differentiate between the two possible interpretations of the effect. One possibility is that the rats in the pentobarbital-beforestimulation group retained the tolerance to ethanol that they had developed in Experiment 2, while the rats in the pentobarbital-after-stimulation group lost theirs because they were stimulated in a drug-free state during the schedule of pentobarbital injections. A second possibility is that both groups of rats lost their tolerance to ethanol's anticonvulsant effect because they were all stimulated in the absence of ethanol, while the rats in the pentobarbitalbefore-stimulation group developed contingent tolerance to pentobarbital that was manifested in the development of contingent cross-tolerance to ethanol.

\section{GENERAL DISCUSSION}

These experiments have shown that the drug-effect contingency plays a role in drug tolerance, cross-tolerance, and the cross maintenance of tolerance. In Experiment 1B, the development of tolerance to the anticonvulsant effect of pentobarbital was shown to be contingent: significantly more tolerance developed in the rats that repeatedly received pentobarbital before the convulsive stimulations than in the rats that received it after. In Experiment 2, contingent tolerance to the anticonvulsant effect of pentobarbital was shown to transfer to the anticonvulsant effect of ethanol: significantly more cross-tolerance was evident in the rats from the pentobarbital-before-stimulation group, which had repeatedly experienced the anticonvulsant effect of pentobarbital. In Experiment 3, tolerance to the anticonvulsant effect of ethanol was maintained in the subjects that were stimulated after each of a series of pentobarbital injections, but it was lost in those that were stimulated prior to each of the pentobarbital injections.

Although the present studies focused on forelimb clonus duration as a measure of seizure severity-largely because it has proven to be more reliable and more sensitive to drug effects than the commonly used motorseizure-class measure (Racine, 1972; Pinel \& Rovner, 1978)-it should be emphasized that we monitored motor seizure class in each experiment and that the statistical 
analysis of motor seizure class confirmed the present conclusions in every way.

A comparison of the results of Experiment $1 \mathrm{~A}$ and $1 \mathrm{~B}$ emphasizes the dangers of concluding, on the basis of experiments involving a limited range of doses or a limited number of injection trials, that tolerance to a particular drug effect does not develop. Although it has been reported that high treatment doses facilitate the development of tolerance to a drug's effects (see Kalant, LeBlanc, \& Gibbins, 1971), in Experiment $1 \mathrm{~A}$ there was no evidence of tolerance development after the rats had received 10 injections of the high treatment dose of pentobarbital (20 mg/kg). In contrast, substantial tolerance was apparent in the pentobarbital-before-stimulation group of Experiment $1 \mathrm{~B}$, when more injections (15) were administered at smaller doses $(15 \mathrm{mg} / \mathrm{kg})$.

Theories of tolerance that fail to recognize the central role played by drug effects, as opposed to mere drug exposure, have difficulty accounting for the observation in Experiment 3 that tolerance to the anticonvulsant effect of ethanol was maintained by a regimen of pentobarbital injections only if those injections produced anticonvulsant effects (contingent cross maintenance of tolerance). However, this finding supports our earlier observation (Mana \& Pinel, 1987) that the key factor in the maintenance of contingent tolerance is not the presence or absence of the drug per se; it is the performance of the criterion response in the presence of the drug. On the basis of this earlier research, we made two successful predictions in Experiment 3: (1) that tolerance to the anticonvulsant effect of ethanol would rapidly decline in rats stimulated every other day in the absence of the drug, despite the fact that they received an injections of pentobarbital $1 \mathrm{~h}$ after each convulsion, and (2) that tolerance to ethanol would not significantly decline in rats receiving pentobarbital injections $1 \mathrm{~h}$ after each bidaily convulsive stimulation. The ability of the drug-effect theory of tolerance to predict these ostensibly anomalous findings is another significant step toward establishing its credibility and generality.

\section{REFERENCES}

Allen, F. D., \& Swinyard, C. A. (1949). Evaluation of tissue tolerance to ethyl alcohol by alterations in electroshock seizure thresholds in rats. Anatomical Records, 103, 419.

Carlton, P. L., \&olgin, D. L. (1971). Contingent tolerance to the anorexigenic effects of amphetamine. Physiology \& Behavior, 7, 221-223.

CHEN, C. S. (1968). A study of the alcohol tolerance effect and an introduction of a new behavioural technique. Psychopharmacologia, 12 , 433-440.

Commissaris, R. L., ReCH, R. H. (1981). Tolerance to pentobarbital and ethanol following chronic pentobarbital administration in the rat. Substance \& Alcohol Actions/Misuse, 2, 331-339.

Demellweek, C., Goudie, A. J. (1983). Behavioural tolerance to amphetamine and other psychostimulants: The case for considering behavioural mechanisms. Psychopharmacology, 80, 287-307.

FreY, H.-H., \& KampMANN, E. (1965). Tolerance to anticonvulsant drugs. Acta Pharmacologica et Toxicologica, 22, 159-171.

Kalant, H., LeBlanc, A. E., \& Gibins, R. J. (1971). Tolerance to, and dependence on some non-opiate psychotropic drugs. Pharmacological Reviews, 23, 135-191.

Kumar, R., \& Stolerman, J. P. (1977). Experimental and clinical aspects of drug dependence. In L. L. Iverson, S. D. Iverson, \& S. H. Snyder (Eds.), Principles of behavioral Pharmacology: Vol. 7 (pp. 321-367). New York: Plenum.

Lê, A. D., El-Ghund, M., Khanna, J. M., Kalant, H. (1986, June). Learning as a factor in the development of tolerance (T) to ethanol $(E)$ and cross tolerance $(X T)$ to pentobarbital $(P B)$ with respect to their motor impairment (MI) effects. Paper presented at the Third Congress of the International Society for Biomedical Research on Alcoholism, Helsinki.

Mana, M. J., \& Pinel, J. P. J. (1987). Response contingency and the dissipation of ethanol tolerance. In K. O. Lindros, R. Ylikahri, \& K. Kiianmaa (Eds.), Advances in biomedical alcohol research (pp. 413-416). Oxford, England: Pergamon Press.

Pellegrino, L. J., Pellegrino, A. S., \&ushman, A. J. (1979). A stereotaxic atlas of the rat brain (2nd ed.). New York: Plenum.

Pinel, J. P. J., Colbourne, B., Sigalet, J. P., \& Renfrey, G. (1983). Learned tolerance to the anticonvulsant effects of alcohol in rats. Pharmacology, Biochemistry, \& Behavior, 18, 507-510.

PineL, J. P. J., \& MANA, M. J. (1986). Kindled seizures and drug tolerance. In J. A. Wada (Ed.), Kindling 3 (pp. 393-407). New York: Raven Press.

Pinel, J. P. J., Mana, M. J., \& Renfrey, G. (1985). Contingent tolerance to the anticonvulsant effects of alcohol. Alcohol, 2, 495-499.

Pinel, J. P. J., \& Rovner, L. I. (1978). Experimental epileptogenesis: Kindling-induced epilepsy in rats. Experimental Neurology, 58, 190-202.

Prichard, J. W. (1980). Phenobarbital: Proposed mechanism of antiepileptic action. In G. H. Glaser, J. K. Penry, \& D. M. Woodbury (Eds.), Antiepileptic drugs: Mechanisms of action (pp. 553-562). New York: Raven Press.

RACTNE, R. J. (1972). Modification of seizure activity by electrical stimulation: II. Motor seizure. Electroencephalography \& Clinical Neurophysiology, 32, 281-294.

Traynor, A. E., Schlapfer, W. T., \& Barondes, S. J. (1980). Stimulation is necessary for the development of tolerance to a neuronal effect of ethanol. Journal of Neurobiology, 11, 633-637.

(Manuscript received March 11, 1988; revision accepted for publication August 20, 1988.) 